\title{
Extension of modified RAND to multiphase flash specifications based on state functions other than $(T, P)$
}

Paterson, Duncan; Michelsen, Michael Locht; Yan, Wei; Stenby, Erling Halfdan

Published in:

Fluid Phase Equilibria

Link to article, DOI:

10.1016/j.fluid.2017.10.019

Publication date:

2017

Document Version

Peer reviewed version

Link back to DTU Orbit

Citation $(A P A)$ :

Paterson, D., Michelsen, M. L., Yan, W., \& Stenby, E. H. (2017). Extension of modified RAND to multiphase flash specifications based on state functions other than (T,P). Fluid Phase Equilibria, 458, 288-299. https://doi.org/10.1016/j.fluid.2017.10.019

\section{General rights}

Copyright and moral rights for the publications made accessible in the public portal are retained by the authors and/or other copyright owners and it is a condition of accessing publications that users recognise and abide by the legal requirements associated with these rights.

- Users may download and print one copy of any publication from the public portal for the purpose of private study or research.

- You may not further distribute the material or use it for any profit-making activity or commercial gain

- You may freely distribute the URL identifying the publication in the public portal 


\title{
Extension of Modified RAND to Multiphase Flash Specifications Based on State Functions Other than $(T, P)$
}

\author{
Duncan Paterson $^{\mathrm{a}}$, Michael L. Michelsen ${ }^{\mathrm{b}}$, Wei Yan ${ }^{\mathrm{a}, *}$, Erling H. Stenby ${ }^{\mathrm{a}}$ \\ ${ }^{a}$ Center for Energy Resources Engineering, Department of Chemistry, Technical University \\ of Denmark, DK-2800 Lyngby, Denmark \\ ${ }^{b}$ Center for Energy Resources Engineering, Department of Chemical and Biochemical \\ Engineering, Technical University of Denmark, DK-2800 Lyngby, Denmark
}

\begin{abstract}
The recently proposed modified RAND formulation is extended from isothermal multiphase flash to several other state function based flash specifications. The obtained general formulation is applicable to chemical equilibrium although this study is focused on flash with only phase equilibrium. It is demonstrated that a common symmetric Jacobian matrix can be formulated for all of these flash specifications. Newton iteration with the common Jacobian is used to converge for the majority of cases and a Q-function maximisation with nested isothermal flash in the inner loop is used for the non-convergent exceptions. For isothermal flash with modified RAND, it can happen in rare occasions that the modified RAND step is ascending in the Gibbs energy. A correction of the step is proposed for such cases to obtain a descent direction without violating the condition used in the derivation of modified RAND. A two-phase example is used to demonstrate that the described method is suitable for $(H, P),(T, V),(S, V)$ and $(U, V)$ flash specifications and a four-phase case is examined in more detail for the difficult $(U, V)$ case. Two- and three-phase examples close to critical regions are used to demonstrate the effectiveness of the correction procedure for the modified RAND step and to show that satisfactory rates of convergence are obtained.
\end{abstract}

\section{Introduction}

Many simulations in the oil, gas and chemical industry require the solution to the phase equilibrium problem. The classical example is the isothermal flash where composition, pressure and temperature are specified. The conventional approach to solve the isothermal flash problem involves stability analysis (Michelsen, 1982a; Baker et al., 1982) and phase split calculation (Michelsen,

\footnotetext{
${ }^{*}$ Corresponding author
} 
1982b). An updated summary of this conventional approach for isothermal flash can be found in Michelsen and Mollerup (2007).

Paterson et al. (2017) recently presented two RAND-based formulations for isothermal multiphase flash. The one using the classical $(T, P)$ based thermodynamics was referred to as modified RAND, it is similar in form to the nonideal RAND of Greiner (1991) based on the Brinkley-NASA-RAND algorithm (Brinkley, 1947; Huff et al., 1951; White et al., 1958). Its counterpart using the $(T, V)$ based thermodynamics is referred to as vol-RAND. The modified RAND method uses the elemental chemical potentials and phase molar amounts (or chemical potentials and phase molar fractions when there are no reactions) as variables. The resulting formulation treats all components in all phases in the same manner and thus avoids the complex book-keeping for calculating the Hessian matrix in the conventional approach. The method is in principle applicable to situations with chemical equilibrium although Paterson et al. (2017) showed its application only to isothermal multiphase equilibrium.

In addition to isobaric, isothermal flash $(T, P)$ there are a number of other important flash specifications. Isobaric isenthalpic flash $(H, P)$ is useful for steady state simulations and has found renewed interest recently for the simulation of heavy oil production (Chempath et al., 2017). The $(U, V)$ based flash is considered by Smejkal and Mikyska (2017) who propose to directly maximise the entropy to solve this problem, this specification is useful for nonisothermal dynamic simulations. A general framework to deal with a range of flash specifications is proposed by Michelsen (1999), where both a Newton method for two-phase calculations and a method utilising a nested isothermal flash are proposed. Chemical reaction equilibrium is commonly discussed at only a given temperature and pressure, with little consideration to other specifications (Smith and Missen, 1982). One example of the $(T, V)$ specification being used is to solve the association terms in more complex equations of state, which is investigated by Heidemann and Prausnitz (1976) and a more general approach is given byMichelsen and Mollerup (2007) who note that to solve the problem at a given $(T, P)$ leads to a constrained problem.

In this study we show that modified RAND can be extended to five other state function based flash specifications (Section 2) in a manner similar to Michelsen (1999). Modified RAND is well suited to multiphase flash calculations and is developed for simultaneous chemical and phase equilibrium calculations. The extension provided here is applicable to simultaneous chemical and phase equilibrium at different flash specifications although our later discussion is focused only on multiphase equilibrium without chemical reactions. All the flash specifications can be solved quadratically using a common Jacobian. Since the problem is no longer minimisation for the other five types of flash specifications, a nested loop with isothermal modified RAND in the inner loop is needed for the rare non-convergent cases.

Another issue that we address here is how to correct modified RAND for isothermal flash to ensure its convergence even for some challenging situations (Section 3). Using the conventional framework a restricted step method is commonly used for the two-phase case (Michelsen and Mollerup, 2007) to ensure 
convergence. The multiphase case is not well scaled and perturbed decomposition routines are often used (Michelsen, 1982b), though some recent work has utilised the popular trust region type methods (Petitfrere and Nichita, 2014). In general, modified RAND monitors the Gibbs energy at each iteration and it is possible to use a line search to find a step corresponding to a decrease in the Gibbs energy. However in some rare cases the modified RAND step may be in an ascent direction where the line search is not suitable. The procedure must be corrected to find a descent direction in the Gibbs energy. We show how to make a correction which is consistent with the modified RAND derivation. The correction minimally impacts the rate of convergence and can be applied without significant additional effort. This will be compared with the conventional restricted step for two-phase problems and demonstrated to be excellent even for a near tri-critical mixture.

\section{State Function Based Flash Specifications}

There are six specifications for flash which can be written as the minimisation of a state function (Michelsen, 1999). These are given in table 1. Many of these are important to some elements of reservoir or process simulation.

Table 1: State function associated with flash specification

\begin{tabular}{c|c|l} 
Flash specification & State function & Example use \\
\hline$(P, T)$ & $G$ & Commonly used for transient simulation \\
$(V, T)$ & $A$ & Storage tank model \\
$(P, H)$ & $-S$ & Steady state flow \\
$(P, S)$ & $H$ & Reversible expansion and compression \\
$(V, U)$ & $-S$ & Unsteady state flow \\
$(V, S)$ & $U$ & None yet but fits the general scheme
\end{tabular}

It was demonstrated by Michelsen (1999) that the last five problems in table 1 could be posed as a maximisation of a Q-function in one or two variables with the isothermal flash nested in an inner loop. It was also demonstrated that, using the conventional isothermal flash framework for two phases, each of the five could be solved using Newton's method (given a suitable initial estimate), with the nested isothermal flash required only in a small number of cases. In a similar manner it is possible to solve the flash problems in table 1 using the modified RAND framework.

The modified RAND framework was presented by Paterson et al. (2017) as an alternative to the non-ideal RAND of Greiner (1991). It was demonstrated to be well suited to solving the phase split problem involving multiple phases. In the presence of chemical reactions the Lagrangian for the constrained minimisation of the Gibbs energy is:

$$
\mathcal{L}(\boldsymbol{n}, \hat{\boldsymbol{\lambda}})=\sum_{j=1}^{F} \sum_{i=1}^{C} n_{i, j} \mu_{i, j}-\sum_{l=1}^{E} \hat{\lambda}_{l}\left(\sum_{i=1}^{C} \mathcal{A}_{l, i}\left(\sum_{j=1}^{F} n_{i, j}\right)-b_{l}\right)
$$


where the chemical potential is defined as

$$
\mu_{i, j}=\mu_{i}^{0}+R T\left(\ln x_{i, j}+\ln \hat{\varphi}_{i, j}+\ln P\right)
$$

The Lagrange multipliers $\left(\hat{\lambda_{l}}\right)$ are equivalent to the equilibrium elemental potentials (Michelsen and Mollerup, 2007). The formula matrix $\mathcal{A}$ is used to relate the components to the elements. The stationarity equations are given by

$$
\frac{\partial \mathcal{L}}{\partial n_{i, j}}=\mu_{i, j}-\sum_{l=1}^{E} \hat{\lambda}_{l} \mathcal{A}_{l, i}=0, \quad \forall i, j
$$

and

$$
\frac{\partial \mathcal{L}}{\partial \hat{\lambda}_{l}}=-\sum_{i=1}^{C} \mathcal{A}_{l, i}\left(\sum_{j=1}^{F} n_{i, j}\right)+b_{l}=0, \quad \forall l
$$

where equation 3 describes the equilibrium condition and equation 4 describes the elemental balance.

In the original derivation for modified RAND, equation 3 was linearised around the current molar amounts with the aim of finding a Jacobian for isothermal flash (Paterson et al., 2017). In this work a general Jacobian for each flash specification given in table 1 is desired. To find this, we first linearise equation 3 around the molar amounts, temperature and pressure then divide by $R T$ :

$$
\frac{\mu_{i, j}}{R T}+\frac{1}{R T} \sum_{k=1}^{C} \frac{\partial \mu_{i, j}}{\partial n_{k, j}} \Delta n_{k, j}+\frac{1}{R T} \frac{\partial \mu_{i, j}}{\partial T} \Delta T+\frac{1}{R T} \frac{\partial \mu_{i, j}}{\partial P} \Delta P-\sum_{l=1}^{E} \lambda_{l} \mathcal{A}_{l, i}=0,
$$

where we have defined $\lambda_{l}$ as the reduced equilibrium elemental potentials. The change in the molar amounts, $\Delta \boldsymbol{n}_{j}$, cannot be immediately isolated because the matrix of composition derivatives of the chemical potential is singular:

$$
\frac{\partial \boldsymbol{\mu}_{j}}{\partial \boldsymbol{n}_{j}} \boldsymbol{n}_{j}=\mathbf{0}, \quad \forall j
$$

with the vector of molar amounts $\boldsymbol{n}_{j}$ in the nullspace of the matrix. This is often referred to as the Gibbs-Duhem equation. Using the definition of the chemical potential, equation 2, we can split the derivative and introduce the matrix $\boldsymbol{m}$ :

$$
m_{i, k, j}=\beta_{j}\left(\frac{1}{R T} \frac{\partial \mu_{i, j}}{\partial n_{k, j}}+1\right)=\frac{\delta_{i, k}}{x_{i, j}}+\beta_{j} \frac{\partial \ln \hat{\varphi}_{i, j}}{\partial n_{k, j}}
$$

Equation 5 in vector-matrix format for phase $j$ is now given by:

$$
\left(\boldsymbol{m}_{j}-\mathbf{1 1}^{T}\right) \Delta \boldsymbol{n}_{j}=\beta_{j}\left(\mathcal{A}^{T} \boldsymbol{\lambda}-\frac{\boldsymbol{\mu}_{j}}{R T}-\boldsymbol{e}_{j} \Delta T-\gamma_{j} \Delta P\right)
$$

for convenience we have used $\mathbf{1 1 ^ { T }}$ to represent the all-ones matrix and defined:

$$
\boldsymbol{e}_{j}=\frac{1}{R T} \frac{\partial \boldsymbol{\mu}_{j}}{\partial T}, \text { and } \gamma_{j}=\frac{1}{R T} \frac{\partial \boldsymbol{\mu}_{j}}{\partial P}
$$


Using the relation $\mathbf{1}^{T} \boldsymbol{n}_{j}=\beta_{j}$, we obtain:

$$
\boldsymbol{m}_{j} \Delta \boldsymbol{n}_{j}=\mathbf{1} \Delta \beta_{j}+\beta_{j}\left(\mathcal{A}^{T} \boldsymbol{\lambda}-\frac{\boldsymbol{\mu}_{j}}{R T}-\boldsymbol{e}_{j} \Delta T-\gamma_{j} \Delta P\right)
$$

From the Gibbs-Duhem equation it is clear that $\boldsymbol{m}_{j} \boldsymbol{x}_{j}=\mathbf{1}$ and $\boldsymbol{M}_{j} \mathbf{1}=\boldsymbol{x}_{j}$, with $\boldsymbol{M}_{j}=\boldsymbol{m}_{j}^{-1}$. These relations are used to isolate the change in the molar amount of each component in each phase:

$$
\Delta \boldsymbol{n}_{j}=\boldsymbol{x}_{j} \Delta \beta_{j}+\beta_{j} \boldsymbol{M}_{j}\left(\mathcal{A}^{T} \boldsymbol{\lambda}-\frac{\boldsymbol{\mu}_{j}}{R T}-\boldsymbol{e}_{j} \Delta T-\gamma_{j} \Delta P\right)
$$

Multiplying equation 10 by $\mathbf{1}^{T}$ from the left we obtain

$$
\Delta \beta_{j}=\mathbf{1}^{T} \Delta \boldsymbol{n}_{j}=\mathbf{1}^{T}\left(\boldsymbol{x}_{j} \Delta \beta_{j}+\beta_{j} \boldsymbol{M}_{j}\left(\mathcal{A}^{T} \boldsymbol{\lambda}-\frac{\boldsymbol{\mu}_{j}}{R T}-\boldsymbol{e}_{j} \Delta T-\gamma_{j} \Delta P\right)\right)
$$

Using the relations $\mathbf{1}^{T} \boldsymbol{x}_{j}=1$, and $\mathbf{1}^{T} \boldsymbol{M}_{j}=\boldsymbol{x}_{j}^{T}$, the $\Delta \beta_{j}$ terms can be eliminated to arrive at:

$$
\boldsymbol{X}^{T}\left(\mathcal{A}^{T} \boldsymbol{\lambda}-\boldsymbol{e}_{j} \Delta T-\gamma_{j} \Delta P\right)=\boldsymbol{g}
$$

with $\boldsymbol{X}=\left(\boldsymbol{x}_{1}, \boldsymbol{x}_{2}, \ldots, \boldsymbol{x}_{F}\right)$. This equation relates the reduced equilibrium elemental potentials $\boldsymbol{\lambda}$ to the reduced molar Gibbs energy of each phase:

$$
g_{j}=\frac{1}{R T} \sum_{i=1}^{C} x_{i, j} \mu_{i, j}
$$

Linearisation of equation 4 yields for each element:

$$
\sum_{i=1}^{C} \mathcal{A}_{l, i}\left(\sum_{j=1}^{F}\left(n_{i, j}+\Delta n_{i, j}\right)\right)-b_{l}=0
$$

Given a composition which meets the elemental balance, equation 14 can be rewritten for all elements as

$$
\mathcal{A}\left(\sum_{j=1}^{F} \Delta \boldsymbol{n}_{j}\right)=\mathbf{0}
$$

Substituting equation 10 into equation 15 we obtain

$$
\begin{aligned}
\mathcal{A}\left(\sum_{j=1}^{F} \beta_{j} \boldsymbol{M}_{j}\right) \mathcal{A}^{T} \boldsymbol{\lambda} & +\mathcal{A} \boldsymbol{X} \Delta \boldsymbol{\beta}-\mathcal{A}\left(\sum_{j=1}^{F} \beta_{j} \boldsymbol{M}_{j} \boldsymbol{e}_{j}\right) \Delta T \\
& -\mathcal{A}\left(\sum_{j=1}^{F} \beta_{j} \boldsymbol{M}_{j} \gamma_{j}\right) \Delta P=\mathcal{A}\left(\sum_{j=1}^{F} \beta_{j} \boldsymbol{M}_{j} \frac{\boldsymbol{\mu}_{j}}{R T}\right)
\end{aligned}
$$


The $F$ equations in 12 with the $E$ equations in 16 relate the $F+E$ variables of reduced equilibrium elemental potentials $\boldsymbol{\lambda}$ and phase fractions $\Delta \boldsymbol{\beta}$ if the temperature and pressure are specified (isothermal, isobaric flash). Once solved the correction for each component in each phase is found from equation 10.

For the remaining specifications defined in table 1 it is necessary to introduce a number of additional constraints. For the $(S, P)$ and $(S, V)$ specifications, the entropy is constrained:

$$
S(T, P, \boldsymbol{n})-S^{s p e c}=0
$$

For the $(H, P)$ specification the enthalpy is constrained:

$$
H(T, P, \boldsymbol{n})-H^{s p e c}=0
$$

for the $(T, V),(S, V)$ and $(U, V)$ the volume is constrained:

$$
V^{\text {spec }}-V(T, P, \boldsymbol{n})=0
$$

Finally for the $(U, V)$ specification the internal energy is constrained:

$$
U(T, P, \boldsymbol{n})-U^{s p e c} \approx H(T, P, \boldsymbol{n})-U^{\text {spec }}-P V^{\text {spec }}
$$

This approximation is exact at the solution and can be safely used.

Each constraint is linearised around the temperature, pressure and molar amount of each component in each phase to obtain:

$$
\begin{gathered}
\frac{S-S^{s p e c}}{R T}+\frac{C_{p}}{R T^{2}} \Delta T-\frac{1}{R T} \frac{\partial V}{\partial T} \Delta P-\sum_{j=1}^{F} \boldsymbol{e}_{j}^{T} \Delta \boldsymbol{n}_{j}=0 \\
\frac{H-H^{s p e c}}{R T^{2}}+\frac{C_{p}}{R T^{2}} \Delta T-\frac{1}{R T}\left(\frac{\partial V}{\partial T}-\frac{V}{T}\right) \Delta P-\sum_{j=1}^{F} \boldsymbol{\xi}_{j}^{T} \Delta \boldsymbol{n}_{j}=0 \\
\frac{V^{s p e c}-V}{R T}-\frac{1}{R T} \frac{\partial V}{\partial T} \Delta T-\frac{1}{R T} \frac{\partial V}{\partial P} \Delta P-\sum_{j=1}^{F} \gamma_{j}^{T} \Delta \boldsymbol{n}_{j}=0 \\
\frac{H-U^{s p e c}-P V^{s p e c}}{R T^{2}}+\frac{C_{p}}{R T^{2}} \Delta T-\frac{1}{R T}\left(\frac{\partial V}{\partial T}+\frac{V^{s p e c}-V}{T}\right) \Delta P-\sum_{j=1}^{F} \boldsymbol{\xi}_{j}^{T} \Delta \boldsymbol{n}_{j}=0
\end{gathered}
$$

where we have used $\boldsymbol{\xi}_{j}=\frac{1}{R} \frac{\partial \frac{\mu_{j}}{T}}{\partial T}$. To arrive at a symmetric matrix for all specifications it is necessary to make a number of simplifications. The term $\xi_{i, j}$ can be split as:

$$
\frac{\partial \frac{\boldsymbol{\mu}_{j}}{T}}{\partial T}=\frac{1}{T} \frac{\partial \boldsymbol{\mu}_{j}}{\partial T}-\frac{\boldsymbol{\mu}_{j}}{T^{2}}
$$

In the summation term in equation $18 \mathrm{~d}$ the second term from the RHS of equation 19 will be:

$$
\sum_{j=1}^{F} \frac{\boldsymbol{\mu}_{j}^{T}}{R T^{2}} \Delta \boldsymbol{n}_{j}
$$


At equilibrium, the reduced chemical potentials of each phase are related to the reduced elemental potentials through:

$$
\frac{\boldsymbol{\mu}_{j}}{R T}=\mathcal{A}^{T} \boldsymbol{\lambda}
$$

The summation, equation 20 , can be safely removed from $18 \mathrm{~d}$ because:

$$
\sum_{j=1}^{F} \frac{\boldsymbol{\mu}_{j}^{T}}{R T^{2}} \Delta \boldsymbol{n}_{j}=\frac{1}{T} \sum_{j=1}^{F}\left(\mathcal{A}^{T} \boldsymbol{\lambda}\right)^{T} \Delta \boldsymbol{n}_{j}=\frac{\boldsymbol{\lambda}^{T}}{T} \mathcal{A}\left(\sum_{j=1}^{F} \Delta \boldsymbol{n}_{j}\right)=0
$$

This is zero because equation 15 must be satisfied given a mixture that meets the elemental balance.

Therefore it is possible to replace $\boldsymbol{\xi}_{j}$ in equations $18 \mathrm{~b}$ and $18 \mathrm{~d}$ with $\boldsymbol{e}_{j}$ for each phase. The pressure derivative in equation $18 \mathrm{~b}$ is not used in any specification and can be dropped. At the solution the volume will be identical to the volume specification, therefore the third term in equation $18 \mathrm{~d}$ can be simplified. The four equations can be rewritten as:

$$
\begin{gathered}
\frac{C_{p}}{R T^{2}} \Delta T-\frac{1}{R T} \frac{\partial V}{\partial T} \Delta P-\sum_{j=1}^{F} \boldsymbol{e}_{j}^{T} \Delta \boldsymbol{n}_{j}=r_{T} \\
-\frac{1}{R T} \frac{\partial V}{\partial T} \Delta T-\frac{1}{R T} \frac{\partial V}{\partial P} \Delta P-\sum_{j=1}^{F} \gamma_{j}^{T} \Delta \boldsymbol{n}_{j}=r_{P}
\end{gathered}
$$

where $r_{T}$ and $r_{P}$ are defined in table 2 .

Table 2: The elements of $r_{T}$ and $r_{P}$ for a given specification

\begin{tabular}{l|l|l} 
Flash specification & $r_{T}$ & $r_{P}$ \\
\hline$(T, P)$ & $-\frac{1}{R T^{2}}\left(H^{\text {spec }}-H\right)$ & - \\
$(H, P)$ & $\frac{1}{R T}\left(S^{\text {spec }}-S\right)$ & - \\
$(S, P)$ & - & $\frac{1}{R T}\left(V-V^{\text {spec }}\right)$ \\
$(T, V)$ & $\frac{1}{R T^{2}}\left(U^{\text {spec }}+P V^{\text {spec }}-H\right)$ & $\frac{1}{R T}\left(V-V^{\text {spec }}\right)$ \\
$(U, V)$ & $\frac{1}{R T}\left(S^{\text {spec }}-S\right)$ & $\frac{1}{R T}\left(V-V^{\text {spec }}\right)$ \\
$(S, V)$ &
\end{tabular}

The change in the molar amount of each component in each phase defined in equation 10 is substituted into equations 22 to obtain:

$$
\begin{aligned}
& -\sum_{j=1}^{F} \beta_{j} \boldsymbol{e}_{j}^{T} \boldsymbol{M}_{j} \mathcal{A}^{T} \boldsymbol{\lambda}-\sum_{j=1}^{F} \boldsymbol{x}_{j}^{T} \boldsymbol{e}_{j} \Delta \beta_{j}+C_{x} \Delta T+\Omega \Delta P=r_{T}-\sum_{j=1}^{F} \beta_{j} \boldsymbol{e}_{j}^{T} \boldsymbol{M}_{j} \frac{\boldsymbol{\mu}_{j}}{R T} \\
& -\sum_{j=1}^{F} \beta_{j} \gamma_{j}^{T} \boldsymbol{M}_{j} \mathcal{A}^{T} \boldsymbol{\lambda}-\sum_{j=1}^{F} \boldsymbol{x}_{j}^{T} \gamma_{j} \Delta \beta_{j}+\Omega \Delta T+\Psi \Delta P=r_{P}-\sum_{j=1}^{F} \beta_{j} \gamma_{j}^{T} \boldsymbol{M}_{j} \frac{\boldsymbol{\mu}_{j}}{R T}
\end{aligned}
$$


Here we have used

$$
\begin{gathered}
C_{x}=\frac{C_{p}}{R T^{2}}+\sum_{j=1}^{F} \beta_{j} \boldsymbol{e}_{j}^{T} \boldsymbol{M}_{j} \boldsymbol{e}_{j} \\
\Omega=\sum_{j=1}^{F} \beta_{j} \boldsymbol{e}_{j}^{T} \boldsymbol{M}_{j} \gamma_{j}-\frac{1}{R T} \frac{\partial V}{\partial T}=\sum_{j=1}^{F} \beta_{j} \gamma_{j}^{T} \boldsymbol{M}_{j} \boldsymbol{e}_{j}-\frac{1}{R T} \frac{\partial V}{\partial T} \\
\Psi=\sum_{j=1}^{F} \beta_{j} \gamma_{j}^{T} \boldsymbol{M}_{j} \gamma_{j}-\frac{1}{R T} \frac{\partial V}{\partial P}
\end{gathered}
$$

A common symmetric matrix can then be set up with the specifications given in table 2 from equations 12, 16, 23 and 24 .

$$
\left(\begin{array}{cccc}
\mathcal{A} \sum_{j=1}^{F} \beta_{j} \boldsymbol{M}_{j} \mathcal{A}^{T} & \boldsymbol{A} \boldsymbol{X} & -\boldsymbol{t} & -\boldsymbol{q} \\
(\boldsymbol{A} \boldsymbol{X})^{T} & \mathbf{0} & \boldsymbol{s}_{1} & \boldsymbol{s}_{2} \\
-\boldsymbol{t}^{T} & \boldsymbol{s}_{1}^{T} & C_{x} & \Omega \\
-\boldsymbol{q}^{T} & \boldsymbol{s}_{2}^{T} & \Omega & \Psi
\end{array}\right)\left(\begin{array}{c}
\boldsymbol{\lambda} \\
\Delta \boldsymbol{\beta} \\
\Delta T \\
\Delta P
\end{array}\right)=\left(\begin{array}{c}
\boldsymbol{u}_{1} \\
\boldsymbol{u}_{2} \\
u_{3} \\
u_{4}
\end{array}\right)
$$

where $\boldsymbol{u}_{1}=\sum_{j=1}^{F} \beta_{j} \boldsymbol{M}_{j} \frac{\boldsymbol{\mu}_{j}}{R T}$

$u_{2, j}=\sum_{i=1}^{C} x_{i, j} \frac{\mu_{i, j}}{R T} \quad \forall j$

$u_{3}=r_{T}-\sum_{j=1}^{F} \beta_{j} \boldsymbol{e}_{j}^{T} \boldsymbol{M}_{j} \frac{\boldsymbol{\mu}_{j}}{R T}$

$u_{4}=r_{P}-\sum_{j=1}^{F} \beta_{j} \gamma_{j}^{T} \boldsymbol{M}_{j} \frac{\boldsymbol{\mu}_{j}}{R T}$

$\boldsymbol{t}=\mathcal{A} \sum_{j=1}^{F} \beta_{j} \boldsymbol{M}_{j} \boldsymbol{e}_{j}$

$\boldsymbol{q}=\mathcal{A} \sum_{j=1}^{F} \beta_{j} \boldsymbol{M}_{j} \boldsymbol{\gamma}_{j}$

$s_{1, j}=-\boldsymbol{x}_{j}^{T} \boldsymbol{e}_{j} \quad \forall j$

$s_{2, j}=-\boldsymbol{x}_{j}^{T} \gamma_{j} \quad \forall j$

This system of equations is solved to find the elemental potentials, change in phase amounts, change in temperature and change in pressure. These can then be used to find the update to the molar amount of each component in each phase from equation 10. The only change made for each flash specification is given in table 2. Only the $(T, P)$ specification has a suitable objective function to minimise (the Gibbs energy). For the other flash specifications, the Newton iteration with proper initial estimates is expected to converge in the majority of cases and a fall back method is needed for the non-convergent situations.

In the absence of chemical reactions it is possible to make a number of simplifications. The elemental feed becomes the component feed $b_{i}=z_{i}$, the formula matrix is the identity matrix $\mathcal{A}=\boldsymbol{I}$ and the reduced chemical potential can be replaced by the fugacity $\ln \hat{\boldsymbol{f}}_{j}$. The matrix in equation 28 is replaced by

$$
\left(\begin{array}{cccc}
\sum_{j=1}^{F} \beta_{j} \boldsymbol{M}_{j} & \boldsymbol{X} & -\boldsymbol{t} & -\boldsymbol{q} \\
\boldsymbol{X}^{T} & \mathbf{0} & \boldsymbol{s}_{1} & \boldsymbol{s}_{2} \\
-\boldsymbol{t}^{T} & \boldsymbol{s}_{1}^{T} & C_{x} & \Omega \\
-\boldsymbol{q}^{T} & \boldsymbol{s}_{2}^{T} & \Omega & \Psi
\end{array}\right)\left(\begin{array}{c}
\boldsymbol{\lambda} \\
\Delta \boldsymbol{\beta} \\
\Delta T \\
\Delta P
\end{array}\right)=\left(\begin{array}{c}
\boldsymbol{u}_{1} \\
\boldsymbol{u}_{2} \\
u_{3} \\
u_{4}
\end{array}\right)
$$


with

$\boldsymbol{u}_{1}=\sum_{j=1}^{F} \beta_{j} \boldsymbol{M}_{j} \ln \hat{\boldsymbol{f}}$

$u_{2, j}=\sum_{i=1}^{C} x_{i, j} \ln \hat{\boldsymbol{f}} \quad \forall j$

$u_{3}=r_{T}-\sum_{j=1}^{F} \beta_{j} \boldsymbol{e}_{j}^{T} \boldsymbol{M}_{j} \ln \hat{\boldsymbol{f}}$

$u_{4}=r_{P}-\sum_{j=1}^{F} \beta_{j} \gamma_{j}^{T} M_{j} \ln \hat{\boldsymbol{f}}$

$\boldsymbol{t}=\sum_{j=1}^{F} \beta_{j} \boldsymbol{M}_{j} \boldsymbol{e}_{j}$

$\boldsymbol{q}=\sum_{j=1}^{F} \beta_{j} \boldsymbol{M}_{j} \gamma_{j}$

$\boldsymbol{e}_{j}=\frac{\partial \ln \hat{\boldsymbol{f}}_{j}}{\partial T}$

$\gamma_{j}=\frac{\partial \ln \hat{\boldsymbol{f}}_{j}}{\partial P}$

This is again a symmetric matrix for any specification.

An ideal solution approximation can be arrived at by assuming that the composition derivatives of the fugacity coefficients are equal to zero. In this case the $\boldsymbol{M}_{j}$ matrix becomes diagonal with elements $x_{i, j}$. Therefore the top left $C \times C$ elements of the matrix are replaced by

$$
\sum_{j=1}^{F} \beta_{j} \boldsymbol{M}_{j}=\boldsymbol{Z}
$$

Instead of factorising the full RAND matrix it is possible to use diagonal term to reduce the size of the system of equations to be solved from $C+F+2$ to $F+2$ (or $F+1$ for $(V, T),(H, P)$ and $(S, P)$ specifications). This can be used as an alternative to the explicit inverse for the two-phase case described by Michelsen (1999) and is suitable for any number of phases.

\section{Correction of the modified RAND step}

For the $(T, P)$ specification it is possible to monitor the convergence of the modified RAND method through the system Gibbs energy. Each step should lead to a reduction in Gibbs energy. In the majority of cases where the modified RAND correction corresponds to a descent direction, the proper stepping can be found through a line search. In the absence of chemical reactions such a descent direction requires:

$$
\sum_{j=1}^{F} \sum_{i=1}^{C} \Delta n_{i, j} \ln \hat{f}_{i, j}<0
$$

During the iteration, if all phases are intrinsically stable (i.e. the Hessian of the Gibbs energy of a phase is not indefinite), the direction will be descending. If one or more phases are intrinsically unstable, the direction may become uphill and requires correction. An uphill direction can be revealed by checking equation 31. In general, such a correction is needed only in near critical regions or some challenging multiphase situations. We describe below the general correction method for multiple phases and a special adaptation to the two-phase case. 
An indefinite $\boldsymbol{M}$ matrix (i.e. unstable phase) will be revealed during the inversion of the $\boldsymbol{m}$ matrix (we drop the phase index in the discussion here for convenience). If one or more $\boldsymbol{m}$ matrix is indefinite then the step generated may be in an ascent direction. It is then necessary to modify the indefinite matrix (or matrices) to correct the direction. However an arbitrary correction to the $\boldsymbol{m}$ matrix will lead to the violation of the requirement that $\boldsymbol{M} \mathbf{1}=\boldsymbol{x}$, which is used to obtain equation 10 . We desire a correction which satisfies this requirement and only minimally hinders the rate of convergence.

To find such a correction we first rewrite matrix $\boldsymbol{m}$ as:

$$
\boldsymbol{m}=\boldsymbol{D}^{-1 / 2} \boldsymbol{Q} \boldsymbol{D}^{-1 / 2}
$$

where $\boldsymbol{D}$ is diagonal with elements $x_{i}$. The elements of $\boldsymbol{Q}$ are given by

$$
Q_{i, k}=\delta_{i, k}+\sqrt{x_{i} x_{k}} \frac{\partial \ln \hat{\varphi}_{i}}{\partial x_{k}}
$$

For an ideal solution, all eigenvalues of this matrix equal 1. At the limit of intrinsic stability exactly one eigenvalue is zero. The case of an indefinite $\boldsymbol{M}$ corresponds to one negative eigenvalue of $\boldsymbol{Q}$.

The numerically smallest eigenvalue of $\boldsymbol{Q}$ can be evaluated using the inverse iteration

$$
\boldsymbol{Q}^{-1} \hat{\boldsymbol{u}}=\frac{1}{\lambda_{\min }} \hat{\boldsymbol{u}}
$$

with $\lambda_{\min }$ the minimum absolute eigenvalue and $\hat{\boldsymbol{u}}$ the corresponding normalised eigenvector. It should be noted we use $\lambda$ to discuss eigenvalues in this section. They should be easily distinguished from the elemental potentials (and reduced elemental potentials) from the context. Equation 34 can be rewritten as

$$
\boldsymbol{D}^{1 / 2} \boldsymbol{Q}^{-1} \boldsymbol{D}^{1 / 2} \boldsymbol{D}^{-1 / 2} \hat{\boldsymbol{u}}=\frac{1}{\lambda_{\min }} \boldsymbol{D} \boldsymbol{D}^{-1 / 2} \hat{\boldsymbol{u}}
$$

or equivalently

$$
\boldsymbol{D}^{-1} \boldsymbol{M} \boldsymbol{w}=\frac{1}{\lambda_{\min }} \boldsymbol{w}, \quad \hat{\boldsymbol{u}}=\boldsymbol{D}^{1 / 2} \boldsymbol{w}
$$

In general the numerically smallest eigenvalue of $\boldsymbol{Q}$ is also negative. To correct for this negative eigenvalue we add to $\boldsymbol{Q}$ the matrix $\epsilon \hat{\boldsymbol{u}} \hat{\boldsymbol{u}}^{T}$, where $\epsilon+\lambda_{\min }=d>$ 0 . Here $d$ is chosen to be a small positive number, e.g. 0.01. This corresponds to adding to $\boldsymbol{Q}^{-1}$ the contribution $k \hat{\boldsymbol{u}} \hat{\boldsymbol{u}}^{T}$ with $k=1 / d-1 / \lambda_{\min }$, and thus adding to $\boldsymbol{M}$ the contribution $k \boldsymbol{v} \boldsymbol{v}^{T}$, where $v_{i}=\hat{u}_{i} \sqrt{x_{i}}$. This update preserves the property $\boldsymbol{M} \mathbf{1}=\boldsymbol{x}$ due to the orthogonality of eigenvectors as both $\hat{\boldsymbol{u}}$ and $\sqrt{\boldsymbol{x}}$ are eigenvectors of $\boldsymbol{Q}$.

As we have already evaluated the matrix $M$ the minimum eigenvalue $\lambda_{\min }$ and corresponding eigenvector $\hat{\boldsymbol{u}}$ are found with minimal effort. Once found the term $k \boldsymbol{v} \boldsymbol{v}^{T}$ can be added to the indefinite matrix $\boldsymbol{M}$ and substituted into the system of equations 29 resulting in:

$$
\left(\begin{array}{cc}
\sum_{j=1}^{F} \beta_{j} \boldsymbol{M}_{j} & \boldsymbol{X} \\
\boldsymbol{X}^{T} & \mathbf{0}
\end{array}\right)\left(\begin{array}{c}
\boldsymbol{\lambda} \\
\Delta \boldsymbol{\beta}
\end{array}\right)=\left(\begin{array}{l}
\boldsymbol{u}_{1} \\
\boldsymbol{u}_{2}
\end{array}\right)+\alpha\left(\begin{array}{c}
\boldsymbol{v} \\
\mathbf{0}
\end{array}\right)
$$


here

$$
\alpha=\beta_{j} k \boldsymbol{v}^{T}\left(\ln \hat{\boldsymbol{f}}_{j}-\boldsymbol{\lambda}\right)
$$

The matrix on the left hand side is already available in a factorised form. The system of equations is solved to find

$$
\left(\begin{array}{c}
\boldsymbol{\lambda} \\
\Delta \boldsymbol{\beta}
\end{array}\right)=\left(\begin{array}{c}
\boldsymbol{\lambda}_{0} \\
\Delta \boldsymbol{\beta}_{0}
\end{array}\right)+\alpha\left(\begin{array}{c}
\boldsymbol{\lambda}_{1} \\
\Delta \boldsymbol{\beta}_{1}
\end{array}\right)
$$

The $\alpha$ term is found from the closing equation

$$
\alpha=\beta_{j} k \boldsymbol{v}^{T}\left(\ln \hat{\boldsymbol{f}}_{j}-\boldsymbol{\lambda}_{0}-\alpha \boldsymbol{\lambda}_{1}\right)
$$

The update to the unstable phase is then calculated as:

$$
\begin{gathered}
\Delta \boldsymbol{x}_{j}=\boldsymbol{M}_{j}\left(\boldsymbol{\lambda}-\ln \hat{\boldsymbol{f}}_{j}\right)+k \boldsymbol{v}^{T}\left(\boldsymbol{\lambda}-\ln \hat{\boldsymbol{f}}_{j}\right) \boldsymbol{v} \\
\Delta \boldsymbol{n}_{j}=\boldsymbol{x}_{j} \Delta \beta_{j}+\beta_{j} \Delta \boldsymbol{x}_{j}
\end{gathered}
$$

For the remaining phases the relation in equation 10 remains unchanged. We note that $\boldsymbol{\lambda}_{0}$ and $\Delta \boldsymbol{\beta}_{0}$ have already been evaluated to determine the direction in equation 31. The proposed update requires only the evaluation of a single eigenvalue, eigenvector pair, no additional matrix factorisations are necessary.

If the numerically smallest eigenvalue of $\boldsymbol{Q}$ is positive additional steps will be necessary to identify the negative eigenvalue. If more than one matrix is indefinite then it may be necessary to correct all indefinite matrices, though the direction from equation 31 can be checked after correcting each indefinite matrix. Such instances are rare in practical applications.

Though this correction is shown for only phase equilibria, the $\boldsymbol{M}$ matrix can in principle be updated in the same way and the procedure modified in a similar manner in the presence of chemical reactions. The update will preserve the property $\boldsymbol{M} \mathbf{1}=\boldsymbol{x}$ and will not require the re-factorisation or inversion of any additional matrices.

\subsection{Two-phase case}

The two-phase case, in the absence of chemical reactions, is a special case of multiphase flash. This can be posed in a simple manner using Newton's method on the Gibbs energy with one phase set as dependent. The molar amount of each component can be defined through the material balance:

$$
n_{i, l}=z_{i}-n_{i, v}
$$

The gradient of the reduced Gibbs energy is then:

$$
f_{i}=\ln \hat{f}_{i, v}-\ln \hat{f}_{i, l}
$$

an element of the Hessian is

$$
H_{i, k}=\frac{1}{\beta_{l}}\left(\frac{\delta_{i, k}}{x_{i}}+\beta_{l} \frac{\partial \ln \hat{\varphi}_{i, l}}{\partial n_{k}}\right)+\frac{1}{\beta_{v}}\left(\frac{\delta_{i, k}}{y_{i}}+\beta_{v} \frac{\partial \ln \hat{\varphi}_{i, v}}{\partial n_{k}}\right)-\frac{1}{\beta_{l} \beta_{v}}
$$


The correction is found from the Newton step

$$
\Delta \boldsymbol{n}_{v}=-\boldsymbol{H}^{-1} \boldsymbol{f}
$$

To avoid round-off errors the update should be applied to the component present in the smaller amount $\left(\Delta n_{i, l}=-\Delta n_{i, v}\right)$.

If the Hessian is not positive definite the direction generated may not be towards a minimum in the Gibbs energy. The restricted step method is commonly used to ensure convergence in the case of a non-positive definite Hessian. The updated positive definite Hessian is given by:

$$
\hat{\boldsymbol{H}}=\boldsymbol{H}+\alpha \boldsymbol{s}
$$

where $s$ is a correction vector added to the diagonal of the matrix, and $\alpha$ is evaluated in a way to ensure that the Hessian is positive definite (e.g. using the method of Hebden (1973)), as recommended by Michelsen and Mollerup (2007). One option for the correction vector is the diagonal contribution to the ideal solution Hessian (which is always positive definite):

$$
s_{i}=\frac{z_{i}}{x_{i, l} x_{i, v}}
$$

An alternative is to use the procedure correction for the modified RAND method. The Hessian in equation 44 can be rewritten

$$
\boldsymbol{H}=\frac{1}{\beta_{l}} \boldsymbol{m}_{l}+\frac{1}{\beta_{v}} \boldsymbol{m}_{v}-\frac{\mathbf{1 1}^{T}}{\beta_{l} \beta_{v}}
$$

with the $\boldsymbol{m}$ matrix defined in equation 7 . The Hessian will only be indefinite if at least one of the $\boldsymbol{m}$ matrices is indefinite.

We assume that $\boldsymbol{m}_{l}$ is indefinite with a single negative eigenvalue. The minimum eigenvalue $\lambda_{\text {min }}$ and corresponding eigenvector $\hat{\boldsymbol{u}}$ of the $\boldsymbol{Q}_{l}$ matrix based on this $\boldsymbol{m}_{l}$ matrix can be evaluated from equation 36 . To correct for the negative eigenvalue we add to $\boldsymbol{m}_{l}$ the correction $k \boldsymbol{v} \boldsymbol{v}^{T}$ with $k+\lambda_{\min } \geq 0$ with

$$
v_{i}=\frac{u_{i}}{x_{i, l}}
$$

An indefinite Hessian reveals itself in the factorisation step, however a new factorisation with the modified Hessian can be avoided. We may write

$$
\boldsymbol{H} \Delta \boldsymbol{n}_{v}=-\boldsymbol{f}-\alpha \boldsymbol{v}
$$

with $\alpha=\frac{k}{\beta_{l}} \boldsymbol{v}^{T} \Delta \boldsymbol{n}_{v}$. Using the factorised Hessian we find

$$
\Delta \boldsymbol{n}_{v}=\Delta \boldsymbol{n}_{v}^{0}-\alpha \Delta \boldsymbol{n}_{v}^{1}
$$

with the closing relation

$$
\alpha=\frac{k}{\beta_{l}} \boldsymbol{v}^{T}\left(\Delta \boldsymbol{n}_{v}^{0}-\alpha \Delta \boldsymbol{n}_{v}^{1}\right)
$$


Usually $k=-\lambda_{\min }$ is an adequate correction. Following the update to $\Delta \boldsymbol{n}_{v}$ the update will be in a descent direction however it may be necessary to use a line search to obtain a decrease in the objective function.

For the two phase case the restricted step method often leads to rapid convergence and is the preferred implementation for isothermal flash (Michelsen and Mollerup, 2007). However for the multiphase case there is no obvious ideal scaling factor for the Hessian. Often perturbed decomposition routines are used to avoid repeating the expensive decomposition (e.g. Murray's method of lines (Fletcher, 1981)). These methods are robust however the rate of convergence can be adversely affected.

\section{Results}

Flash calculation with state function based specifications other than $(T, P)$ are more complex to solve than the conventional isothermal flash. We adopt the solution strategy recommended by Michelsen (1999) where a Newton approach is used to handle the majority of cases and a Q-function maximisation is used as a fall back method. Michelsen (1999) demonstrated the strategy only for two-phase cases using the conventional flash formulation. Here, we apply it to general multiphase equilibrium and use the modified RAND formulation. The solution strategy adapted to the modified RAND formulation is as follows:

1. The initial estimate is generated using the Wilson K-factor approximation

$$
\hat{\varphi}_{i, v}=1, \quad \hat{\varphi}_{i, l}=\frac{P_{c, i}}{P} \ln \left(5.373\left(1+\omega_{i}\right)\left(1-\frac{T_{c, i}}{T}\right)\right)
$$

The temperature and pressure are found by solving the Wilson equation with its temperature and pressure derivatives to meet the specified constraints.

2. The ideal solution approximation is used with the modified RAND method for two steps to improve the initial estimate. The system of equations to be solved, equation 29 is reduced in size from $C+F+2$ to $F+2$ using the explicit inverse of part of the matrix.

3. The full modified RAND, equation 29, is used to converge the system of equations until the deviation from the specified constraints and fugacity of each component differed by less than $10^{-10}$.

4. Once converged stability analysis is used to check if an additional phase can be introduced. If a new phase can be introduced then the composition from stability analysis is used in zero amount and returned to step 2.

5. If the total number of iterations (where each iteration is counted as the decomposition of the full RAND matrix, equation 28) exceeds $10 \times(F+$ 1) then Q-function maximisation (Michelsen, 1999) is used to find the solution. This is also used when the pressure or temperature are oscillating to extremely large or negative values. The Q-function maximisation uses isothermal modified RAND to solve the phase split calculation and carries out stability analysis to identify the global minimum in the Gibbs energy. 
Three examples are considered. A summary is given in table 3. Example one has a maximum of two phases and is considered as a typical two-phase example. Example two is a complex five-component mixture with up to four phases in equilibrium, this is more complex than most typical mixtures. Example three is a three-component mixture which is considered close to its tri-critical point and used to show that the correction to the modified RAND matrix is excellent even for such complex flash calculations. Details on each mixture are given in the appendix Appendix A.1.

Table 3: Summary of the mixtures considered in this work

\begin{tabular}{l|l|l|l}
\hline & Components & Max phases & System description \\
\hline Example 1 & 7 & 2 & natural gas \\
Example 2 & 5 & 4 & $\mathrm{C}_{1}, \mathrm{C}_{2}, \mathrm{C}_{3}, \mathrm{CO}_{2} \mathrm{H}_{2} \mathrm{~S}$ \\
Example 3 & 3 & 3 & $\mathrm{C}_{1}, \mathrm{C}_{2}, \mathrm{n}-\mathrm{C}_{8}$ \\
\hline
\end{tabular}

For example 1 a window of specifications between $110 \mathrm{~K}<T<300 \mathrm{~K}$ and 1 bar $<P<100$ bar was scanned with an interval of $0.1 \mathrm{~K}$ and 0.1 bar. At each $(T, P)$ specification the solution to the flash problem was found using isothermal modified RAND. The number of iterations necessary to solve the $(T, P)$ flash problem is given in figure 1a, the maximum number of iterations for this mixture is 12 when using modified RAND. At the solution each of the possible specifications was recorded $(U, V, S$ and $H)$. The problem was then re-initialised with the $(S, P)(H, P),(T, V),(S, V)$ and $(U, V)$ specifications then the solution strategy given above was carried out. This allows for a fair comparison of each of the flash specifications since the solution conditions are all the same. The number of iterations was recorded, with one iteration counted as one factorisation of the full modified RAND matrix, equation 29 (not the ideal solution simplification). The number of iterations necessary for each of the other flash specifications did not exceed 30 (where a switch is made to Q-function maximisation) and are plotted on figure 1.

The computational cost relative to $(T, P)$ flash of $(H, P),(S, P),(T, V)$, $(S, V)$ and $(U, V)$ flash was approximately $1.20,1.17,1.21,1.44$, and 1.44 respectively. A switch to Q-function maximisation in the two-phase region was necessary in $0.007 \%, 0.004 \%, 0.002 \%, 0.553 \%$ and $0.182 \%$ of cases respectively for each flash specification. The specifications $(U, V)$ and $(S, V)$ are clearly significantly more difficult than the other specifications, however it is still rare to require the backup nested loop method. Over the full phase envelope the number of iterations is relatively small and the computational cost not significantly greater than isothermal flash. Close to the critical point all of the methods have difficulties as there is not an objective function to minimise, and therefore oversteps often lead to failures. The corresponding figures mapped onto the state function variable space are given in appendix Appendix A.2.

To demonstrate the multiphase capabilities of the modified RAND approach the $(U, V)$ flash was used for a more complex five-component mixture (example 


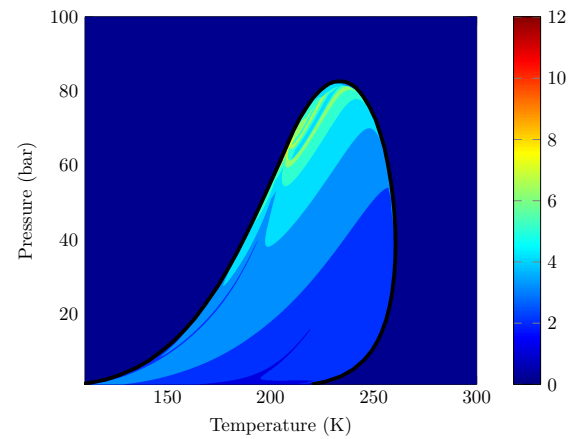

(a) $(P, T)$ Flash

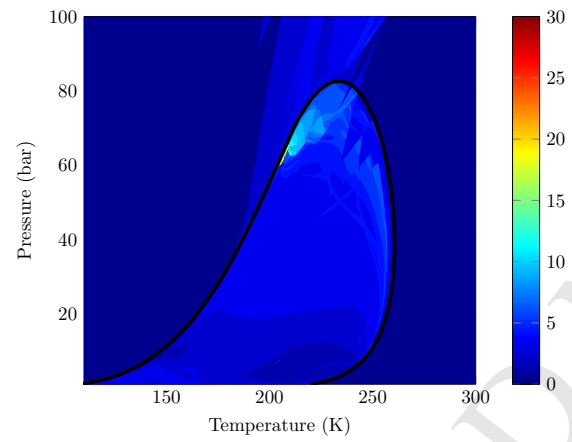

(c) $(P, H)$ Flash

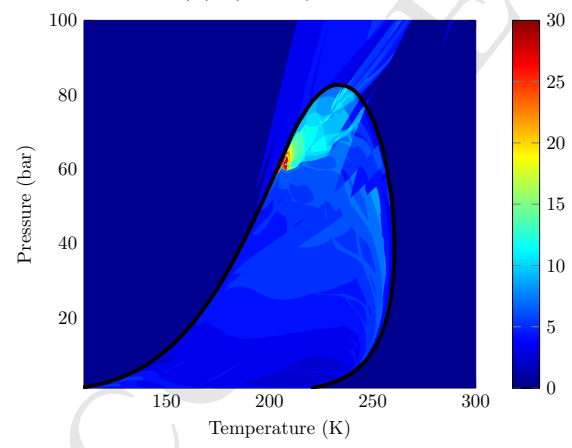

(e) $(V, S)$ Flash

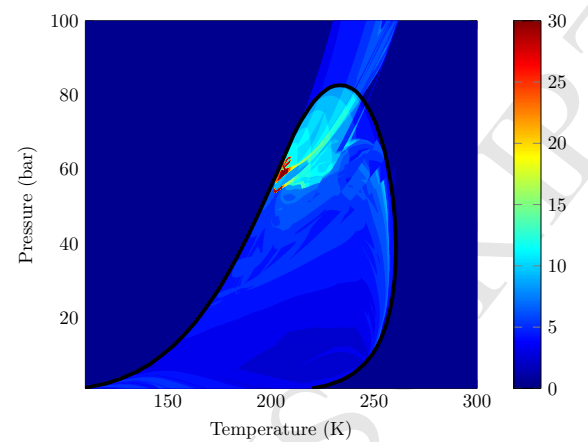

(b) $(V, T)$ Flash

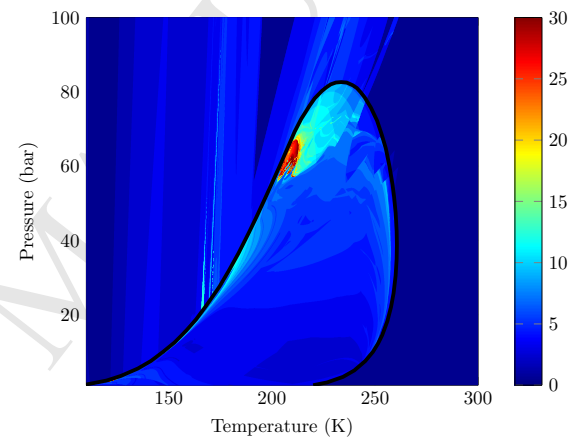

(d) $(P, S)$ Flash

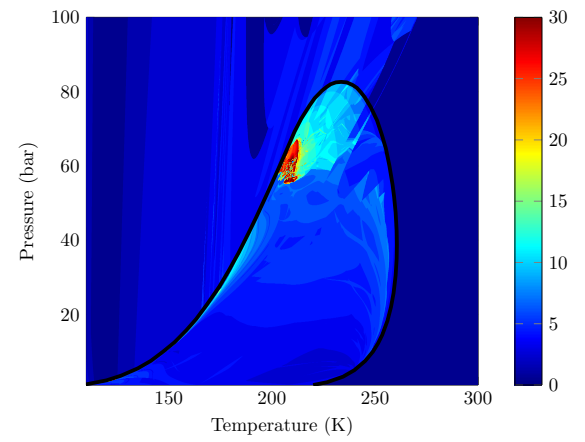

(f) $(V, U)$ Flash

Figure 1: Modified RAND iterations to solve a range of flash problem specifications for example 1, a 7-component natural gas mixture. One iteration is counted as the factorisation of the full modified RAND matrix. 
two). A region of $130 \mathrm{~K}<T<300 \mathrm{~K}$ and $1 \mathrm{bar}<P<100 \mathrm{bar}$ was scanned using the $(T, P)$ specifications. At the solution to the isothermal flash, the internal energy and volume were evaluated and the flash re-initialised,. The number of iterations necessary to solve the $(U, V)$ flash is shown in figure 2 in the same manner as for figure 1.

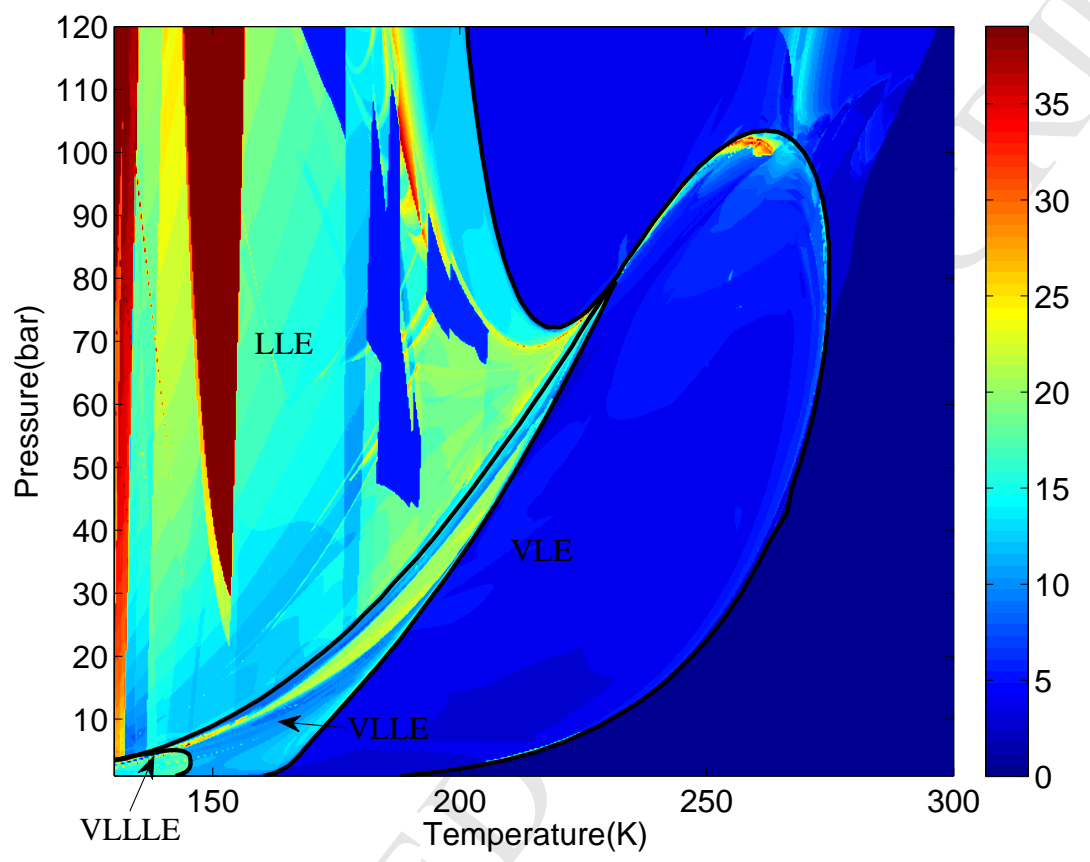

Figure 2: Number of iterations to solve the UV flash phase split calculation for a complex 5-component mixture, with up to 4 phases where the three liquids are either methane rich, hydrogen sulphide rich or carbon dioxide rich.

For example 2 there are difficulties in the LLE region at low temperatures and high pressures. This is in part due to the difficulty to match the volume with $(U, V)$ flash often leading to temperatures outwith the bounds of the ideal gas heat capacity equation, or leading to poor initial estimates which need more iterations to converge. Only $3 \%$ of cases required a switch to Q-function maximisation. The total computational time taken for $(U, V)$ flash was 2 times the time taken for $(P, T)$ flash.

Even in the most complex 4 phase region the method is reasonably robust. Figure 3 shows the number of iterations necessary in this region and the dotted region shows the approximate region where Q-function maximisation was necessary. The limit of iterations was set at 30 before switching to Q-function maximisation for this figure.

Even in the four phase region convergence is generally obtained in a reasonable number of iterations as shown in figure 3 , with only $8 \%$ of cases using the backup Q-function maximisation. The total cost of $(U, V)$ flash was only 


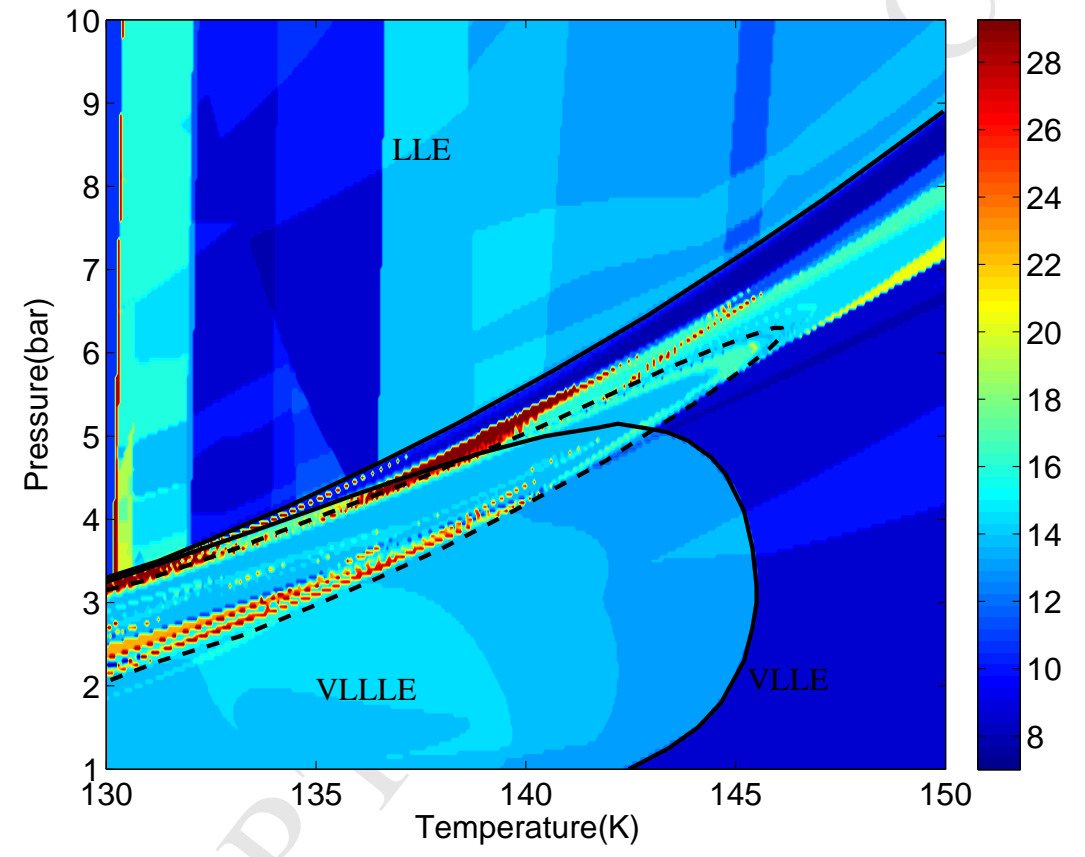

Figure 3: Phase envelope for a complex 5-component mixture with 4 phases, showing the number of iterations to obtain convergence in the 4 phase region. Dotted region shows approximate region where Q-function maximisation was necessary. 
2.3 times that of $(T, P)$ flash in this region. The intermediate solutions for three-phase and four-phase examples are presented in table 4.

Table 4: $(U, V)$ flash specifications and intermediate solutions in $\mathrm{T}(\mathrm{K})$ and $\mathrm{P}$ (bar) for five component mixture, example 2. Also compares the number of iterations to the $(P, T)$ specification at the solution conditions.

\begin{tabular}{|c|c|c|c|c|c|c|c|c|c|c|}
\hline \multicolumn{2}{|c|}{ Conditions } & \multicolumn{2}{|c|}{ K-Wilson } & \multicolumn{2}{|c|}{ 2-phase } & \multicolumn{2}{|c|}{ 3-phase } & 4-phase & \multicolumn{2}{|c|}{ Iterations } \\
\hline$\frac{U}{R}(\mathrm{~K})$ & $V\left(\frac{1}{\mathrm{~mol}}\right)$ & $\mathrm{T}$ & $\mathrm{P}$ & $\mathrm{T}$ & $\mathrm{P}$ & $\mathrm{T}$ & $\mathrm{P}$ & $\mathrm{T} \quad \mathrm{P}$ & $(U, V)$ & $(P, T)$ \\
\hline-1492.7 & 6.939 & 132.5 & 0.91 & 122.0 & 0.93 & 127.2 & 0.97 & 130 & 11 & 11 \\
\hline-1408.9 & 7.802 & 144.5 & 0.97 & 137.3 & 0.98 & 139.2 & 0.99 & 140 & 13 & 13 \\
\hline-1471.7 & 2.322 & 151.1 & 2.95 & 133.3 & 2.85 & 139.1 & 2.96 & 140 & 14 & 11 \\
\hline-1691.9 & 0.360 & 162.9 & 9.90 & 144.4 & 7.06 & 150 & 8.5 & 3-phase & 7 & 6 \\
\hline
\end{tabular}

The initial estimate generated by the Wilson K-factor approximation is often in close agreement with the final temperature of the mixture. The largest deviation from the final temperature for the conditions presented in table 4 was $13 \mathrm{~K}$ and 1.5bar. The intermediate solutions may move further from the final mixture temperature as the non-idealities are taken into account.

Although it is rare for one phase to be unstable (i.e. for the Hessian of the Gibbs energy of that phase to be indefinite) during flash calculations, it is possible with poor initialisation or if the mixture is close to the critical point. Example 1 with a temperature of $202.997 \mathrm{~K}$ and pressure of $58.7 \mathrm{bar}$ is very close to the critical point as demonstrated on figure 1. The procedure correction for the modified RAND framework is compared to the restricted step method using the ideal solution correction vector at these conditions. Following stability analysis of the feed mixture a trial phase at a minimum in the tangent plane distance is identified. Two steps of successive substitution are taken before switching to the second order method with the phase fraction and maximum difference between component fugacities shown in figure 4 .

After two steps of successive substitution, the incipient phase has a phase fraction of $\beta=0.008$. The direction of the step is evaluated using equation 31 . This is found as

$$
\sum_{j=1}^{F} \sum_{i=1}^{C} \Delta n_{i, j} \ln \hat{f}_{i, j}=4 \times 10^{-6}
$$

Since this is greater than 0 , a line search alone will not yield a decrease in the Gibbs energy. Correction to the modified RAND step is necessary. The minimum absolute eigenvalue for the Hessian of the two-phase mixture, equation 44 , was $-1 \times 10^{-7}$.

In this case the phase present in the greater amount is indefinite. The smallest eigenvalue of the $\boldsymbol{Q}$ matrix is $\lambda_{\min }=-0.0121$. Using this with a value of $d=0.01$ we find $\alpha=0.021$ from equation 51 . The change to the equilibrium chemical potential and the change in the flow of each component in the incipient phase, before and after correction, is shown in table 5 .

Before the modified RAND step is corrected, the change in the molar amounts of each component indicates that the phase present in a smaller amount should 


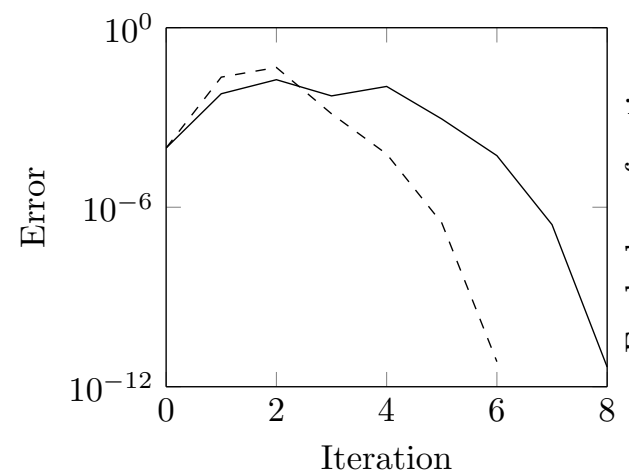

(a) Plot of fugacity error

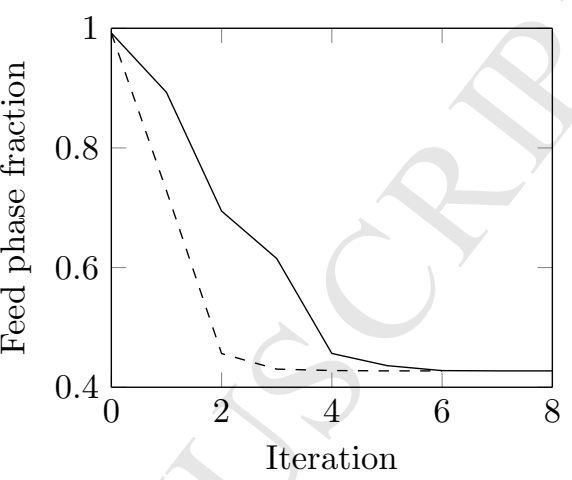

(b) Plot of phase fraction

Figure 4: Comparison of the convergence of a near critical 7-component natural gas mixture, example 1, using the correction to the modified RAND method presented here (dashed) and an restricted step implementation (solid). The error is the maximum absolute difference between the fugacity of a components in the two phases.

Table 5: Equilibrium chemical potential and change in the incipient phase flow before and after correction of the modified RAND step.

\begin{tabular}{l|l|l|l|l} 
Component & \multicolumn{2}{|c|}{$\boldsymbol{\lambda}$} & \multicolumn{2}{c}{$\Delta \boldsymbol{n}$} \\
\hline methane & 3.6095 & 3.6095 & -0.5553 & 0.2502 \\
ethane & -1.6647 & -1.6564 & -0.0138 & 0.0062 \\
propane & -4.3428 & -4.3376 & -0.0034 & 0.0015 \\
n-butane & -6.1390 & -6.1444 & -0.0020 & 0.0009 \\
n-pentane & -8.1262 & -8.1488 & -0.0010 & 0.0005 \\
n-hexane & -10.5054 & -10.5507 & -0.0003 & 0.0002 \\
nitrogen & 0.2717 & 0.2608 & -0.0089 & 0.0040
\end{tabular}


be removed (returning to the trivial solution). Following correction of the modified RAND step the phase fraction increases from $\beta=0.008$ to $\beta=0.271$, the final solution phase fraction is $\beta=0.573$.

In the presented example, figure 4 , which has been described in detail the use of the correction to the modified RAND method requires less iterations than the use of the restricted step method. Similar excellent results are obtained for more complex multiphase examples such as that shown in figure 5 .

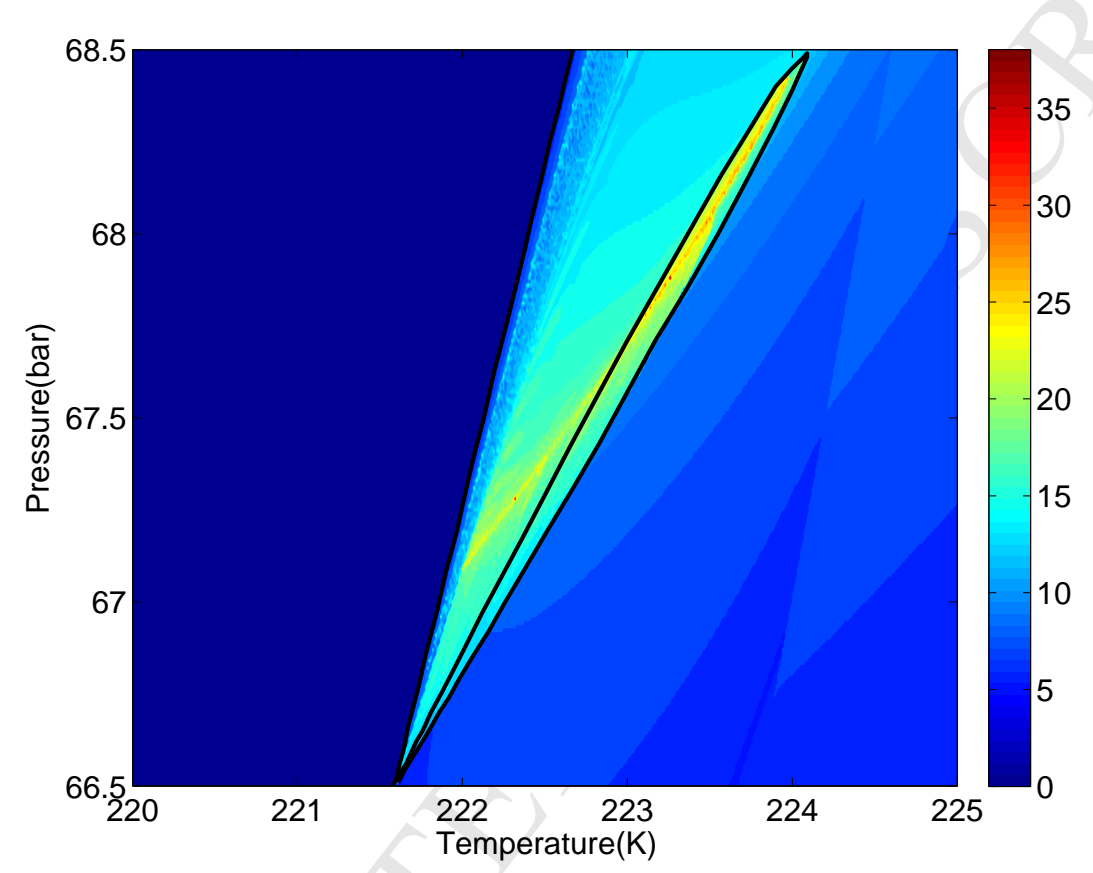

Figure 5: Number of RAND iterations to obtain convergence close to the tri-critical point for mixture of methane, ethane and n-octane, example 3. Three phase contained region inside of the black line and single phase region present to the left hand side.

Example 3 is modelled with $0 k_{i j}$ using SRK where the predicted tricritical point is at molar compositions of $C_{8}=0.64 \%, C_{2}=21.8 \%$ at a temperature of $225.5 \mathrm{~K}$, and a pressure of 69.5bar (Michelsen and Heidemann, 1988). The given composition is $C_{8}=1 \%$, and $C_{2}=21.8 \%$ leading to a small, nearly critical, three-phase region. The number of iterations for the modified RAND approach (where the modified RAND step is often corrected) is given in figure 5. Even in this complex region the number of iterations does not exceed 39. In the same region the number of iterations using the conventional approach (restricted step for two phases and Murray's method of lines for more than two phases) often exceeds 100 with the same convergence criteria. 


\section{Conclusions}

The modified RAND formulation has been extended from the $(T, P)$ specification to five other state function based flash specifications. Only the $(T, P)$ specifications is a minimisation where an objective function (the Gibbs energy) can be checked at each step. The other specifications can be solved by Newton's method using a common Jacobian matrix. In the majority of cases, using a suitable initialisation, it is possible to find the solution rapidly. A switch to Q-function maximisation may be necessary in a small number of cases.

Using the modified RAND method for isothermal flash in the close to critical region may generate an ascent direction in the Gibbs energy. A method to correct the direction has been presented. This correction maintains the properties of the matrix used in the derivation while correcting the direction of the step. The method can be used without an additional factorisation while maintaining the excellent convergence properties of the second order modified RAND method. This has been presented for a close to critical two-phase mixture and a three component mixture close to the tri-critical point. The proposed procedure is compared with the state of the art methods used for isothermal flash calculations

\section{Acknowledgements}

This research was carried out at the Center for Energy Resources Engineering at the Technical University of Denmark. The work was sponsored by ExxonMobil and ConocoPhilips and their contribution is gratefully acknowledged.

\section{Nomenclature}

Non-bolded symbols represent scalars. A bold lowercase symbol represents a column vector while a bold uppercase symbol represents a matrix unless otherwise stated in the text.

\section{Greek symbols}

$\alpha \quad$ Function used as a closing equation defined in equations 39 and 51

$\beta \quad$ Phase molar amount

$\gamma \quad$ Pressure derivative of the reduced chemical potential

$\hat{\lambda}$ Lagrangian multiplier or the equilibrium elemental potential

$\hat{\varphi} \quad$ Fugacity coefficient

$\lambda \quad$ Reduced equilibrium elemental potentials $\hat{\lambda}=\frac{\lambda}{R T}$

$\lambda_{\min } \quad$ Smallest absolute eigenvalue 


$\begin{array}{ll}\mu & \text { Chemical potential } \\ \Omega & \text { Defined in equation } 26 \\ \omega & \text { Acentric factor } \\ \Psi & \text { Defined in equation } 27 \\ \xi & \text { Term defined as } \boldsymbol{\xi}_{j}=\frac{1}{R} \frac{\partial \frac{\mu_{j}}{T}}{\partial T}\end{array}$

\section{Roman symbols}

$\mathcal{A} \quad$ Formula matrix relating components and elements through reactions

$D \quad$ Diagonal matrix with elements $x_{i}$

$\boldsymbol{H} \quad$ Hessian

$M \quad$ Inverse of $\boldsymbol{m}$

$\boldsymbol{m} \quad$ Defined in equation 7

$\boldsymbol{Q} \quad$ Matrix with elements defined in equation 33

$\boldsymbol{v} \quad$ Vector defined as $\boldsymbol{v}=\boldsymbol{D}^{1 / 2} \hat{\boldsymbol{u}}$

$\boldsymbol{w} \quad$ Vector defined as $\boldsymbol{w}=\boldsymbol{D}^{-1 / 2} \hat{\boldsymbol{u}}$

$\boldsymbol{X} \quad$ Matrix of component mole fractions for each phase

$\boldsymbol{Z} \quad$ Diagonal matrix with elements $z_{i}$

$\hat{\boldsymbol{u}} \quad$ Eigenvector

$\hat{f} \quad$ Fugacity

$\mathcal{L} \quad$ Lagrangian

A Helmholtz energy

$b \quad$ Feed element mole number

C Number of components

$C_{p} \quad$ Heat capacity

$C_{x} \quad$ Defined in equation 25

E Number of elements

$e \quad$ Reduced temperature derivative of the chemical potential

F $\quad$ Number of phases 


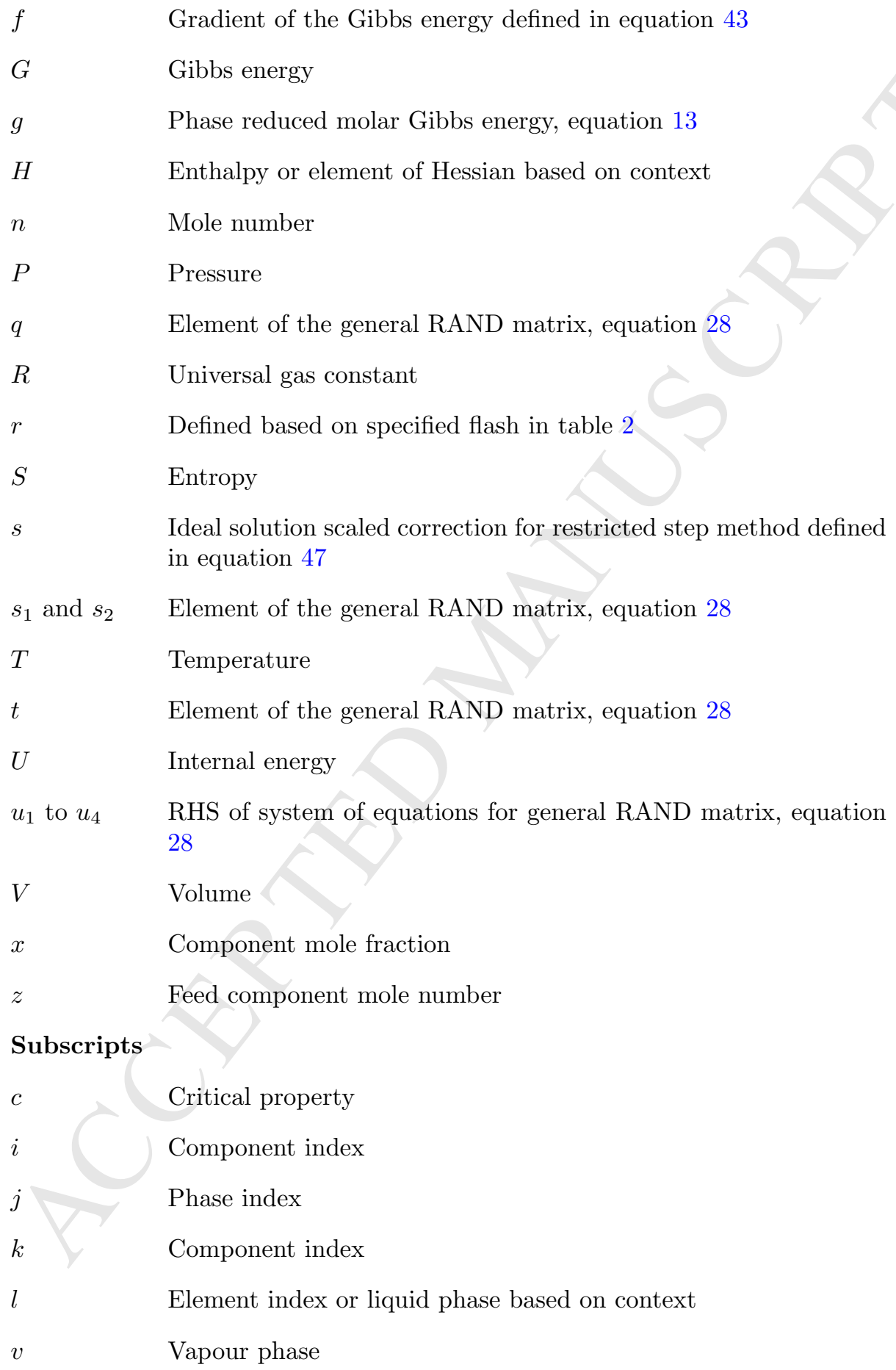




\section{References}

Michael L. Michelsen. The isothermal flash problem. part i. stability. Fluid Phase Equilibria, 9(1):1 - 19, 1982a. ISSN 0378-3812. doi: http://dx.doi.org/ 10.1016/0378-3812(82)85001-2.

Lee E Baker, Alan C Pierce, and Kraemer D Luks. Gibbs Energy Analysis of Phase Equilibria. Spe, (October):1-12, 1982. ISSN 0197-7520. doi: 10.2118/ 9806-PA.

M.L. Michelsen. The isothermal flash problem. part ii. phase split calculation. Fluid Phase Equilibria, 9:21-40, 1982b.

Michael L. Michelsen and Jorgen M. Mollerup. Thermodynamic Models: Fundamentals $\& 3$ Computational Aspects. Tie-Line publications, 2nd edition, 2007. ISBN 87-989961-3-4.

D. Paterson, M. L. Michelsen, E. H. Stenby, and W. Yan. New Formulations for Isothermal Multiphase Flash. In SPE Reservoir Simulation Conference. Society of Petroleum Engineers, feb 2017. ISBN 978-1-61399-483-2. doi: 10.2118/182706-MS.

H. Greiner. An efficient implementation of newton's method for complex nonideal chemical equilibria. Computers \& Chemical Engineering, 15:115 - 123, 1991. ISSN 0098-1354. doi: http://dx.doi.org/10.1016/0098-1354(91)87010-7.

Stuart R. Brinkley. Calculation of the Equilibrium Composition of Systems of Many Constituents. The Journal of Chemical Physics, 15(2):107, 1947. ISSN 00219606. doi: 10.1063/1.1746420.

Vearl N Huff, Sanford Gordon, and Virginia E Morrell. General method and thermodynamic tables for computation of equilibrium composition and temperature of chemical reactions. Technical Report NACA-TR-1037, 1951.

W. B. White, S. M. Johnson, and G. B. Dantzig. Chemical Equilibrium in Complex Mixtures. The Journal of Chemical Physics, 28(5):751-755, 1958. ISSN 0021-9606. doi: 10.1063/1.1744264.

Shaji Chempath, Huafei Sun, and Kjetil Haugen. Optimization Based Isenthalpic Flash for Thermal Reservoir Simulations. In SPE Reservoir Simulation Conference. Society of Petroleum Engineers, feb 2017. ISBN 978-161399-483-2. doi: 10.2118/182702-MS.

Tomas Smejkal and Jiri Mikyska. Phase stability testing and phase equilibrium calculation at specified internal energy, volume, and moles. Fluid Phase Equilibria, 431:82-96, jan 2017. ISSN 0378-3812. doi: 10.1016/J.FLUID.2016.09. 025 .

Michael L. Michelsen. State function based flash specifications. Fluid Phase Equilibria, 158160(0):617 - 626, 1999. ISSN 0378-3812. doi: http://dx.doi. org/10.1016/S0378-3812(99)00092-8. 
William Robert Smith and Ronald William Missen. Chemical Reaction Equilibrium Analysis: Theory and Algorithms. Wiley, 1st editio edition, 1982. ISBN 0471093475 .

R A Heidemann and J M Prausnitz. A van der Waals-type equation of state for fluids with associating molecules. Proceedings of the National Academy of Sciences of the United States of America, 73(6):1773-1776, jun 1976. ISSN 0027-8424. doi: 10.1073/pnas.73.6.1773.

Martin Petitfrere and Dan Vladimir Nichita. Robust and efficient Trust-Region based stability analysis and multiphase flash calculations. Fluid Phase Equilibria, 362:51-68, jan 2014. ISSN 03783812. doi: 10.1016/j.fluid.2013.08.039.

M.D. Hebden. An algorithm for Minimization Using Exact Second Derivatives. Technical report, Atomic Energy Research Estabilishment, Harwell, England, Report No TP 515, 1973.

R. (Roger) Fletcher. Practical methods of optimization. J. Wiley, 1981. ISBN 0471278289 .

Michael L. Michelsen and Robert A. Heidemann. Calculation of tri-critical points. Fluid Phase Equilibria, 39(1):53-74, jan 1988. ISSN 03783812. doi: 10.1016/0378-3812(88)80003-7.

\section{Appendix A. Appendices}

Appendix A.1. Examples used in this work

The three examples considered in this work are presented in tables Appendix A.1, Appendix A.2 and Appendix A.3. All of the examples used the SRK equation of state. The ideal gas heat capacity correlation was:

$$
C_{p}=C_{1}+C_{2}\left(\frac{C_{3}}{T \sinh \left(\frac{C_{3}}{T}\right)}\right)^{2}+C_{4}\left(\frac{C_{5}}{T \cosh \left(\frac{C_{5}}{T}\right)}\right)^{2}
$$

with units of $\mathrm{J} /(\mathrm{kmol} \mathrm{K})$. The parameters used are from the DIPPR database. 
Table Appendix A.1: Properties of 7-component natural gas mixture, example 1

\begin{tabular}{l|l|l|l|l|l}
\hline Component & $\mathrm{z}_{\mathrm{i}}$ & $\mathrm{P}_{\mathrm{c}}(\mathrm{bar})$ & $\mathrm{T}_{\mathrm{c}}(\mathrm{K})$ & $\omega$ & nitrogen $\mathrm{k}_{\mathrm{ij}}$ \\
\hline methane & 0.943 & 45.99 & 190.56 & 0.0115 & 0.02 \\
ethane & 0.027 & 48.72 & 305.32 & 0.0995 & 0.06 \\
propane & 0.0074 & 42.479 & 369.83 & 0.1523 & 0.08 \\
n-butane & 0.0049 & 37.96 & 425.12 & 0.2002 & 0.08 \\
n-pentane & 0.0027 & 33.7 & 469.7 & 0.2515 & 0.08 \\
n-hexane & 0.001 & 30.25 & 507.6 & 0.3013 & 0.08 \\
nitrogen & 0.014 & 34 & 126.2 & 0.0377 & 0 \\
\hline
\end{tabular}

Table Appendix A.2: Properties of 5-component mixture, example 2

\begin{tabular}{l|l|l|l|l|l|l}
\hline Component & $\mathrm{z}_{\mathrm{i}}$ & $\mathrm{P}_{\mathrm{c}}(\mathrm{bar})$ & $\mathrm{T}_{\mathrm{c}}(\mathrm{K})$ & $\omega$ & $\mathrm{CO}_{2} \mathrm{k}_{\mathrm{ij}}$ & $\mathrm{H}_{2} \mathrm{~S} \mathrm{k}_{\mathrm{ij}}$ \\
\hline methane & 0.66 & 45.99 & 190.56 & 0.0115 & 0.12 & 0.08 \\
ethane & 0.03 & 48.72 & 305.32 & 0.0995 & 0.15 & 0.07 \\
propane & 0.01 & 42.479 & 369.83 & 0.1523 & 0.15 & 0.07 \\
$\mathrm{CO}_{2}$ & 0.05 & 73.37 & 304.12 & 0.225 & 0.0 & 0.12 \\
$\mathrm{H}_{2} \mathrm{~S}$ & 0.25 & 90.0 & 373.1 & 0.10 & 0.12 & 0.0 \\
\hline
\end{tabular}

Table Appendix A.3: Properties of 3 component mixture, example 3. All $k_{i j}=0$

\begin{tabular}{l|l|l|l|l}
\hline Component & $\mathrm{z}_{\mathrm{i}}$ & $\mathrm{P}_{\mathrm{c}}(\mathrm{bar})$ & $\mathrm{T}_{\mathrm{c}}(\mathrm{K})$ & $\omega$ \\
\hline methane & 0.772 & 45.99 & 190.56 & 0.0115 \\
ethane & 0.218 & 48.72 & 305.32 & 0.0995 \\
n-octane & 0.01 & 24.90 & 568.7 & 0.3996 \\
\hline
\end{tabular}


Appendix A.2. Mapping of solution to example 1 to the state function variable space

Figure 1 shows the number of iterations to find the solution to the various state function based flash specifications for the six possible flash specifications mapped on to the $(T, P)$ space. It is possible to map the solutions on to the relevant state function variable space as is shown in figure Appendix A.1. 


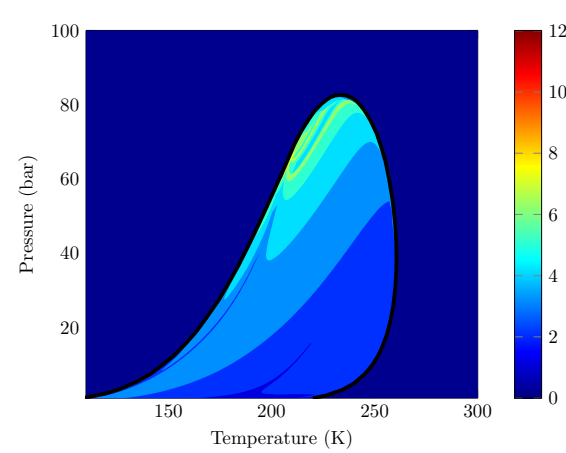

(a) $(P, T)$ Flash

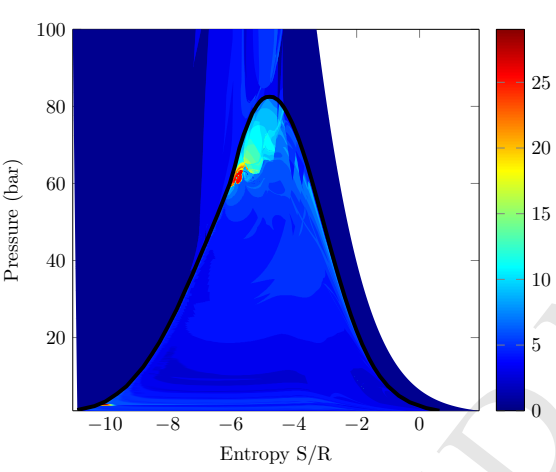

(c) $(P, H)$ Flash

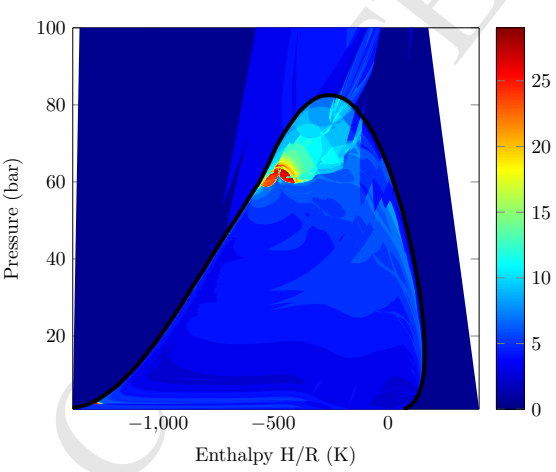

(e) $(V, S)$ Flash

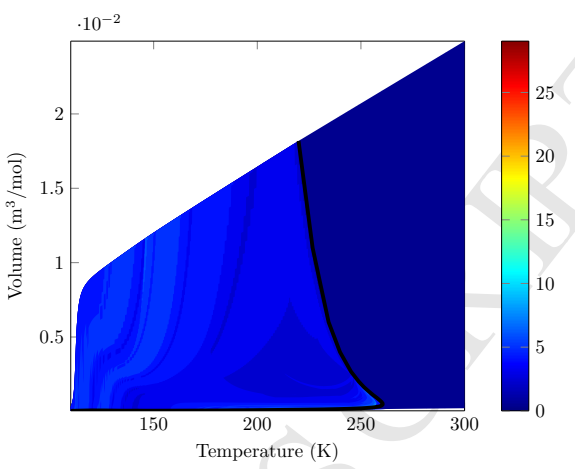

(b) $(V, T)$ Flash

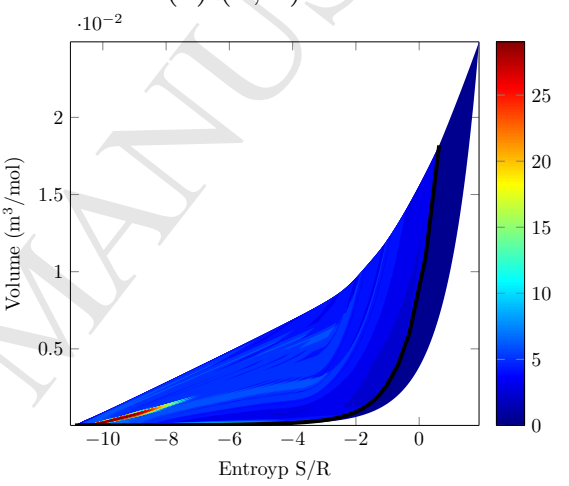

(d) $(P, S)$ Flash

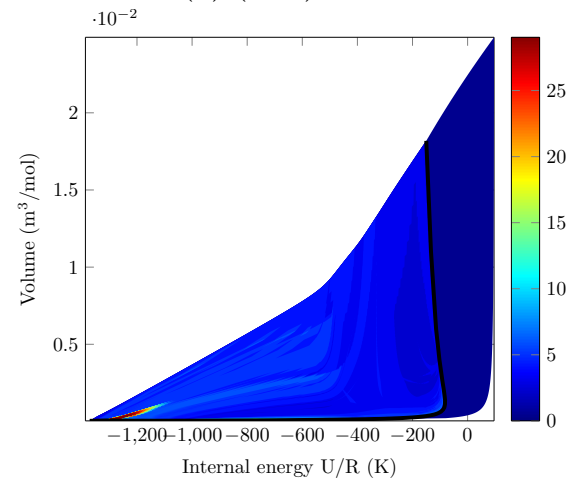

(f) $(V, U)$ Flash

Figure Appendix A.1: Modified RAND iterations to solve a range of flash problem specifications for example 1, a 7-component natural gas mixture. Solution mapped onto the relevant variable space. 